\title{
Editorial \\ Special issue on control theory and technologies in honor of the 70th birthday of Professor Frank L. Lewis
}

It is our great pleasure to put up this special issue in Control Theory and Technology in honor of the 70th birthday of Professor Frank L. Lewis, who has made many truly outstanding contributions to the field of systems and control through the years, which includes applied optimal control, geometric systems theory and nonlinear control in his earlier research period, and his recent focus on cooperative multi-agent distributed systems, reinforcement learning in control, wireless sensor networks for area security monitoring and condition-based maintenance, robotic system control, and manufacturing process control and scheduling to name a few. Featured in this special issue are 10 manuscripts on control theory and technologies, of which many are from the personal and academic friends of Professor Frank Lewis.

Dr. Lewis obtained his Bachelor's degree in Physics and Electrical Engineering and Master's degree in Electrical Engineering at Rice University in 1971. He then spent six years in the U.S. Navy, serving as Navigator and Executive Officer and Acting Commanding Officer. In 1977, he received the Master of Science in Aeronautical Engineering from the University of West Florida. In 1981, he obtained his Ph.D. at Georgia Institute of Technology, where he was employed as a professor from 1981 to 1990 . He is currently a professor of Electrical Engineering at the University of Texas at Arlington (UTA), where he was awarded the Moncrief-O'Donnell Endowed Chair in 1990 at the Automation \& Robotics Research Institute (ARRI). Dr. Lewis is the author of numerous U.S. patents, journal articles and books. He served/serves on many editorial boards. He received Fulbright Research Award 1988, American Society of Engineering Education F. E. Terman Award 1989, International Neural Network Society Gabor Award 2009, U.K. Institute of Measurement and Control Honeywell Field Engineering Medal 2009, 3 Sigma Xi Research Awards, UTA Halliburton Engineering Research Award, UTA Distinguished Research Award, ARRI Patent Awards, and various best paper awards. He was listed in Ft. Worth Business Press Top 200 Leaders in Manufacturing, and was appointed to NAE Committee on Space Station in 1995 and IEEE Control Systems Society Board of Governors in 1996. In 1998, Dr. Lewis was appointed an IEEE Control Systems Society Distinguished Lecturer. He is Fellow of the IEEE, Fellow of IFAC, Fellow of the U.K. Institute of Measurement \& Control, Fellow of US National Academy of Inventors, and Fellow of American Association for the Advancement of Science (AAAS).

We summarize in the following the key contributions of each manuscript included in this special issue.

1) A new semi-tensor product of matrices by Daizhan CHENG, Zequn LIU

Similar to the classical semi-tensor product (STP-I), a new matrix product, called the second semi-tensor product (STP-II) of matrices is proposed in this paper with all its fundamental properties being presented. The equivalence relation caused by STP-II is obtained. Using such an equivalence, a quotient space is also developed with all its vector space structure, metric and metric topology, projection and subspaces being investigated in detail.

(c) 2019 South China University of Technology, Academy of Mathematics and Systems Science, CAS and Springer-Verlag GmbH Germany, part of Springer Nature 
2) Precedence-constrained path planning of messenger UAV for air-ground coordination by Yulong DING, Bin XIN, Jie CHEN

This paper addresses an unmanned aerial vehicle (UAV) path planning problem for a team of cooperating heterogeneous vehicles composed of one UAV and multiple unmanned ground vehicles (UGVs). The UGVs are used as mobile actuators and scattered in a large area. To achieve multi-UGV communication and collaboration, the UAV serves as a messenger to fly over all task points to collect task information for UGVs. The work aims to find the shortest route enabling the UAV to fly over all task points and deliver information to all requested UGVs.

3) Prediction method for energy consumption per ton of fused magnesium furnaces using data driven and mechanism model by Dan GUO, Zhiwei WU, Tianyou CHAI, Jie YANG, Jinliang DING

Electric energy consumed in every ton of acceptable product, namely energy consumption per ton (ECT), is an important overall index for the production process of a fused magnesium furnace. The furnace is the equipment for producing the fused magnesia. The ECT value depends on the current in smelting process. The optimal operation for a fused magnesium furnace is supposed to have the ECT as low as possible, where the key is to predict ECT accurately. By introducing an unknown high-order nonlinear term, this paper builds a dynamic ECT model for different production batches. The simulation experiments are conducted by the on-site data, and the results verify the effectiveness of the proposed prediction method.

4) Distributed adaptive Kalman filter based on variational Bayesian technique by Chen HU, Xiaoming HU, Yiguang HONG

In this paper, distributed Kalman filter design is studied for linear dynamics with unknown measurement noise variance, which modeled by Wishart distribution. To solve the problem in a multi-agent network, a distributed adaptive Kalman filter is proposed with the help of variational Bayesian, where the posterior distribution of joint state and noise variance is approximated by a free-form distribution. The convergence of the proposed algorithm is proved. A distributed target tracking problem is investigated with simulations for illustration.

5) Axis-coupled trajectory generation for chains of integrators through smoothing splines by Shupeng LAl, Menglu LAN, Kehong GONG, Ben M. CHEN

This work presents an axis-coupled trajectory generation algorithm for chains of integrators with an arbitrary order. Special attention has been given to problems with pre-existing nominal plans, which are common in robotic applications. It also handles various type of constraints that can be satisfied on an entire time interval, including nonconvex ones which can be transformed into a series of convex constraints through time segmentation. The proposed method is tested in real flight experiments using quadrotors, which represent high-order integrator systems.

6) An output-based distributed observer and its application to the cooperative linear output regulation problem by Tao LIU, Jie HUANG

An extension of an existing stability result for a class of linear switched systems is obtained. This extended result has relaxed the existence conditions of the output-based distributed observer for a leader system subject to jointly connected switching communication networks in the literature. As an application, the result is utilized to solve the cooperative output regulation problem of a linear multi-agent system subject to jointly connected switching communication networks by composing a purely decentralized control law and the output-based distributed observer based on the certainty equivalence principle.

7) Adaptive dynamic programming for finite-horizon optimal control of linear time-varying discrete-time systems by Bo PANG, Tao BIAN, Zhong-Ping JIANG

This paper studies data-driven learning-based methods for the finite-horizon optimal control of linear time-varying discrete-time systems. A novel finite-horizon policy iteration (PI) method for linear time-varying discrete-time systems is presented. Both data-driven off-policy $\mathrm{PI}$ and value iteration algorithms are derived to find approximate optimal controllers when the system dynamics is completely unknown. The effectiveness and feasibility of the developed methods are successfully validated by a practical example of spacecraft attitude control.

8) Distributed optimal consensus of multiple double integrators under bounded velocity and acceleration by 


\section{Zhirong QIU, Lihua XIE, Yiguang HONG}

A distributed optimal consensus problem for multiple double integrators under bounded velocity and acceleration is investigated. Assigned with an individual and private convex cost which is dependent on the position, each agent needs to achieve consensus at the optimum of the aggregate cost under bounded velocity and acceleration. Based on relative positions and velocities to neighbor agents, a distributed control law is designed by including the integration feedback of position and velocity errors. The result still holds when the relative velocity is not available.

9) Event-triggered state estimation for T-S fuzzy affine systems based on piecewise Lyapunov-Krasovskii functionals by Meng WANG, Jianbin QIU, Gang FENG

This paper investigates the problem of event-triggered $\mathrm{H}_{\infty}$ state estimation for Takagi-Sugeno (T-S) fuzzy affine systems. The objective is to design an event-triggered scheme and an observer such that the resulting estimation error system is asymptotically stable with a prescribed $\mathrm{H}_{\infty}$ performance and at the same time unnecessary output measurement transmission can be reduced. An algorithm is proposed to co-design the observer gains and the eventtriggering parameters to guarantee that the estimation error system is asymptotically stable with a given disturbance attenuation level and the signal transmission rate is reduced as much as possible.

10) Stabilization of discrete-time linear systems by delay independent truncated predictor feedback by Yusheng WEI, Zongli LIN

For a discrete-time linear system with input delay, a truncated predictor feedback law stabilizes the system in the presence of an arbitrarily large delay if the open loop poles are located inside or on the unit circle and the feedback gain is designed by the low gain feedback design technique. A delay independent truncated feedback law results when the delay dependent transition matrix in the truncated predictor feedback law is further discarded. This paper first constructs an example to show that the delay independent truncated predictor feedback law cannot compensate too large a delay if the open loop system has poles on the unit circle at $z \neq 1$. A delay bound is then provided for the stabilizability of a general linear system by the delay independent truncated predictor feedback.

\section{Guest editors:}

Jie HUANG

E-mail: jhuang@mae.cuhk.edu.hk

Ben M. CHEN

E-mail: bmchen@cuhk.edu.hk 